\title{
Comment on 'The Royal College of Ophthalmologists Guidelines on retinal vein occlusions: executive summary'
}

Eye (2016) 30, 771-772; doi:10.1038/eye.2016.63; published online 8 April 2016

I was interested to read 'The Royal College of Ophthalmologists Guidelines on retinal vein occlusions: executive summary'. ${ }^{1}$ I have investigated various aspects of all retinal vein occlusions by prospective and multifaceted studies in large cohorts of patients. Based on my studies, I find several misleading statements in the guidelines, which can have serious impact on the understanding and management of central retinal vein occlusion (CRVO). Following are some examples.

\section{Site of occlusion in CRVO}

Correct information about this is essential in understanding the clinical presentation, complications, and management of CRVO. The Guidelines perpetuate the common misconception about it by stating that it is at the lamina cribrosa. I have discussed at length elsewhere the various reasons why this is not valid. ${ }^{2}$ Very briefly, this belief is based on the site found on histopathology of most of the enucleated eyes for severe and painful neovascular glaucoma, which is a complication of only ischaemic CRVO. But in a study of 620 consecutive CRVO eyes, I found only $20 \%$ had ischaemic CRVO; ${ }^{3}$ in another study of 239 ischaemic CRVO eyes, maximum risk of developing neovascular glaucoma was $34 \%{ }^{4}$ - so the overall incidence of neovascular glaucoma in all CRVO eyes is about $7 \%$. In my studies of over 1000 CRVO eyes, only about $2 \%$ were enucleated because of severe and painful neovascular glaucoma. So findings from enucleated eyes are quite unrepresentative.
Much more reliable information is provided by fluorescein angiography, which always shows retinal blood flow in all CRVO eyes, indicating that the site of occlusion is almost always posterior to the lamina cribrosa. (If the site of occlusion were at the lamina cribrosa, the retinal blood outflow would be completely blocked-if the blood cannot get out, it cannot get in, as is the case in rare eyes where CRVO is associated with secondary central retinal artery occlusion). The severity of CRVO depends upon the distance of the site of occlusion posterior to the lamina cribrosa.

\section{Criteria to differentiate ischaemic from nonischaemic CRVO}

This is the most important issue in CRVO, because the visual outcome, complications, and management of the two types of CRVO are totally different. The Guidelines perpetuate the common misconception of '>10 disc areas of capillary nonperfusion' as the criterion for diagnosis of ischaemic CRVO. I discussed at length elsewhere why that criterion is not at all valid in that differentiation. ${ }^{2}$ I investigated various criteria to differentiate the two types of CRVO, ${ }^{2}$ and showed that fluorescein fundus angiography provided unreliable information about retinal capillary nonperfusion in at least one-third of CRVO eyes during the acute phase, and also that 10 disc areas of capillary nonperfusion did occur in some nonischaemic CRVO eyes.

\section{Natural history of visual outcome in retinal vein occlusion}

This is the most important issue in management; natural history must not be interpreted as the outcome of treatment. I investigated this prospectively 
in 697 eyes with CRVO, ${ }^{5} 67$ with hemi-CRVO (HCRVO), ${ }^{6}$ and 216 with branch retinal vein occlusion (BRVO). ${ }^{7}$ My natural history studies do not support the statements in the Guidelines. In CRVO, the final visual acuity was $6 / 30$ or better in $83 \%$ (better than $6 / 12$ in $61 \%$ ) with nonischaemic CRVO and $6 / 30$ or better in only $12 \%$ with ischaemic CRVO $(P<0.0001)$, and visual field defects were minimal or mild in 95 and $18 \%$, respectively $(P<0.0001)$. In nonischaemic HCRVO eyes, improvement was in $50 \%$ of those with initial visual acuity of $6 / 19$ or worse. In BRVO, in eyes with initial visual acuity of $6 / 19$ or worse, it improved in $69 \%$ for major BRVO and in 53\% for macular BRVO.

\section{Conversion of nonischaemic to ischaemic CRVO}

The Guidelines state that this occurs in $30 \%$. In my study of 500 consecutive nonischaemic CRVO eyes, it was only $12.6 \%{ }^{3}$

\section{Incidence of development of retinal vein occlusion in the fellow eye}

My findings ${ }^{3}$ in 500 consecutive nonischaemic CRVO and 375 consecutive BRVO eyes do not support the information in the Guidelines. The second eye was involved in $7.7 \%$ in nonischaemic CRVO and in 6.6\% in BRVO.

\section{Panretinal photocoagulation (PRP) in ischaemic CRVO}

The justification for this is to prevent the development of ocular neovascularisation (NV) and neovascular glaucoma, which occur only in ischaemic CRVO. The findings of the Central Vein Occlusion Study (CVOS) group are considered the gold standard. $\mathrm{I}^{8}$ pointed out serious flaws in that study. In CVOS study, both the PRP and control groups had a mixture of nonischaemic and ischaemic CRVO eyes, and all eyes that developed 2'clock iris NV were lasered, with no control. I investigated the incidence of various types of $\mathrm{NV}$ in 239 consecutive ischaemic CRVO eyes; ${ }^{4}$ iris NV developed in $52 \%$ and neovascular glaucoma in $34 \%$. So one-third of the eyes in the CVOS did not actually need PRP. Those serious flaws invalidate their conclusion. The authors of CVOS, 9 in response, agreed that those flaws were present. Moreover, in my study of PRP in ischaemic CRVO, ${ }^{10}$ the only significant finding was a marked loss of peripheral visual fields with PRP compared with control eyes; that, combined with universal large central scotoma in these eyes, almost blinded them.

\section{Conflict of interest}

The author declares no conflict of interest.

\section{References}

1 Sivprasad S, Amoaku WM, Hykin PRVO Guideline Group. The Royal College of Ophthalmologists Guidelines on retinal vein occlusions: executive summary. Eye 2015; 29: 1633-1638.

2 Hayreh SS. Prevalent misconceptions about acute retinal vascular occlusive disorders. Prog Retin Eye Res 2005; 24: 493-519.

3 Hayreh SS, Zimmerman MB, Podhajsky P. Incidence of various types of retinal vein occlusion and their recurrence and demographic characteristics. Am J Ophthalmol 1994; 117: 429-441.

4 Hayreh SS, Zimmerman MB. Ocular neovascularization associated with central and hemi-central retinal vein occlusion. Retina 2012; 32: 1553-1565.

5 Hayreh SS, Podhajsky PA, Zimmerman MB. Natural history of visual outcome in central retinal vein occlusion. Ophthalmology 2011; 118: 119-133.

6 Hayreh SS, Zimmerman MB. Hemi-central retinal vein occlusion: natural history of visual outcome. Retina 2012; 32: 68-76.

7 Hayreh SS, Zimmerman MB. Branch retinal vein occlusion: natural history of visual outcome. JAMA Ophthalmol 2014; 132: $13-22$.

8 Hayreh SS. The CVOS Group M and N reports. Ophthalmology 1996; 103: 350-352.

9 Clarkson JG, Coscas G, Finkelstein D, Gutman FAQ, Hillis A, Klein MI et al. The CVOS Group M and N reports. Ophthalmology 1996; 103: 353-354.

10 Hayreh SS, Klugman MR, Podhajsky P, Servais GE, Perkins ES. Argon laser panretinal photocoagulation in ischemic central retinal vein occlusion-a 10-year prospective study. Graefes Arch Clin Exp Ophthalmol 1990; 228: 281-296.

SS Hayreh

Department of Ophthalmology and Visual Sciences, College of Medicine, University of Iowa, Iowa City,

IA, USA

Correspondence: SS Hayreh, Department of Ophthalmology and Visual Sciences, College of Medicine, University of Iowa, 200 Dr Hawkins, Iowa City, IA 52242-1091, USA Tel: +1 319356 2947; Fax: +1 3193537699 . E-mail: Sohan-hayreh@uiowa.edu 